\title{
Estudio retrospectivo:
}

principales causas de ingreso del orden Strigiformes en el Parque Zoológico y Jardín Botánico Nacional Simón Bolívar, San José, Costa Rica durante los años 1995-2015

\author{
Retrospective study: \\ Causes of Strigiformes admission at Parque Zoológico y Jardín Botánico Nacional Simón \\ Bolívar, San José, Costa Rica during 1995-2015
}

\section{Estudo retrospectivo: \\ Principais causas de admissão de admissão de animais da ordem Strigiformes no Parque Zoológico e Jardim Botânico Nacional Simón Bolívar, San José, Costa Rica durante os anos 1995-2015}

\author{
Randall Arguedas ${ }^{1} \bowtie$, Lizbeth Ovares ${ }^{1}$
}

1* Parque Zoológico y Jardín Botánico Nacional Simón Bolívar, Barrio Amón, Calle 13, San José. Apdo.: 11594-1000. Autor para correspondencia ranarg@gmail.com

1 Parque Zoológico y Jardín Botánico Nacional Simón Bolívar, Barrio Amón, Calle 13, San José. Apdo.: 11594-1000. liz.ovares@gmail.com

Recibido: 27 de agosto de 2018 Corregido: 24 de enero de 2019 Aceptado: 12 de febrero de 2019

Resumen: Las poblaciones de rapaces, en la que se incluye al orden Strigiformes, han disminuido en número y se encuentran amenazadas o en peligro de extinción, no solo por la destrucción del hábitat, sino también por las actividades humanas. Los objetivos del presente estudio fueron describir las causas principales de ingreso para las especies del Orden Strigiformes en el Parque Zoológico y Jardín Botánico Nacional Simón Bolívar, así como resumir los diagnósticos clínicos primarios, localidades de procedencia y especies ingresadas, empleando la información generada en las bitácoras de admisión, durante un período de 20 años (1995-2015). De las hojas de ingreso se utilizaron los siguientes datos: fecha de recepción, especie, localidad y motivo. Las causas de admisión se categorizaron en: (1) trauma, (2) huérfanos, (3) intoxicación, (4) electrocución, (5) causas desconocidas. Y la categoría de trauma se clasificó según el tipo de lesión: (1) fractura en extremidades (anteriores o posteriores), (2) trauma cráneo encefálico (TCE), (3) heridas en piel. Se encontró 352 individuos de nueve especies de Strigiformes, al ser la lechucita Neotropical (Megascops choliba) la especie más abundante con 141 individuos (40,05 \%). El mayor porcentaje de estos provino de la provincia de San José $(80,91 \%$, $\mathrm{n}=285$ ). La tendencia de las admisiones de individuos por año, en general se mostró relativamente homogénea en un rango de 10 a 20 individuos, excepto los años $1999(n=35)$ y $2000(n=48)$. El trauma fue la causa de admisión más frecuente, representando el $54 \%(\mathrm{n}=190)$, donde un $78 \%(\mathrm{n}=148)$ corresponde a fracturas. La información generada sobre las especies ingresadas a los sitios de manejo ex situ de fauna silvestre, resulta primordial, ya que existen considerables vacíos de información para muchas especies en particular para los Strigiformes de Costa Rica.

Palabras claves: búhos, fracturas, zoológicos, Costa Rica, Strigiformes 


\begin{abstract}
Raptor populations, including the order Strigiformes, have declined in number and are threatened or endangered, not only by habitat destruction, but also by human activities. The objectives of present study were to describe the main causes of entry for the species of the Order Strigiformes in the Simón Bolívar National Zoological Park and Botanical Garden, as well as to summarize the primary clinical diagnoses, localities of origin and species entered, using the information generated in the records of admission during a period of 20 years (1995-2015). The following data were used from the entry sheets: date of entry, species, locality, and cause: (1) trauma, (2) orphans, (3) intoxication, (4) electrocution, (5) unknown causes. And the category of trauma was classified according to the type of injury: (1) fracture in extremities (anterior or posterior), (2) Brain head trauma (TCE), (3) Skin wounds. There were 352 individuals of nine species of Strigiformes, being the Neotropical owl (Megascops choliba) the most abundant species with 141 individuals $(40.05 \%)$. The highest percentage of individuals came from the province of San José $(80.91 \%, n=285)$. The trend of admissions per year was generally relatively homogeneous in a range of 10 to 20 individuals, except for $1999(\mathrm{n}=35)$ and $2000(\mathrm{n}=48)$. Trauma was the most frequent cause of admission, representing 54\% $(\mathrm{n}=190)$, where $78 \%(\mathrm{n}=148)$ corresponded to fractures. The information generated on the species entered into ex situ wildlife management sites is essential because there are considerable gaps in information for many species, especially for the Costa Rican Strigiformes.
\end{abstract}

Keywords: owls, fractures, zoos, Costa Rica, Strigiformes

Resumo: As populações de aves de rapina, pertencentes a ordem Strigiformes, têm diminuído e estão ameaçadas ou em perigo de extinção, não somente pela destruição do habitat, mas também pelas atividades humanas. Os objetivos deste estudo foram descrever os principais motivos de ingresso das espécies da Ordem Strigiformes no Zoológico e Jardim Botânico Nacional Simón Bolívar; assim como, resumir os diagnósticos clínicos primários, locais de origem e espécies registradas, usando as informações geradas nos registros de admissão, em um período de 20 anos (1995-2015). Os seguintes dados foram utilizados a partir dos formulários de admissão: data de recebimento, espécie, localização e motivo. As causas de ingresso foram categorizadas em: (1) trauma, (2) órfãos, (3) intoxicação, (4) eletrocussão, (5) causas desconhecidas. A categoria de trauma foi classificada de acordo com o tipo de lesão: (1) fratura nas extremidades (anterior ou posterior), (2) traumatismo crânio-encefálico (TCE) e (3) feridas na pele. Foram encontrados 352 indivíduos de nove espécies de Strigiformes, sendo a coruja Neotropical (Megascops choliba) a espécie mais abundante com 141 indivíduos $(40,05 \%)$. A maior porcentagem dos exemplares provenientes da província de San José $(80,91 \%, \mathrm{n}=285)$. A tendência das admissões de indivíduos por ano, em geral, foi relativamente homogênea em um intervalo de 10 a 20 indivíduos, com exceção dos anos de 1999 ( $n=35)$ e 2000 $(\mathrm{n}=48)$. O trauma foi a causa mais frequente de admissão, representando 54\% ( $\mathrm{n}=190)$, sendo que $78 \%(\mathrm{n}=148)$ corresponderam a fraturas. As informações geradas sobre as espécies admitidas nos sítios de manejo ex situ da fauna silvestre são essenciais, pois existem consideráveis lacunas de informação para muitas espécies, em particular para os Strigiformes da Costa Rica.

Palavras-chave: Corujas, fraturas, zoológicos, Costa Rica, Strigiformes

\title{
Introducción
}

Las poblaciones de rapaces, en la que se incluye al orden Strigiformes, han disminuido en número y se encuentran amenazadas o en peligro de extinción, no solo por la destrucción del hábitat, sino también por las actividades humanas (Fix \& Barrows 1990; Deem et al. 1998; Molina-López et al. 2011), debido a esto es que se ha incrementado el interés por las causas de morbilidad de las rapaces de vida libre (Morishita et al. 1998). 
Rev. Ciencias Veterinarias, Vol. 37, Nº 1, [1-11], E-ISSN: 2215-4507, enero-junio, 2019

DOI: http://dx.doi.org/10.15359/rcv.37-1.1

Ciencias

URL: http://www.revistas.una.ac.cr/index.php/veterinaria/index

Asimismo, los estudios de morbilidad complementan la comprensión de los riesgos para las aves rapaces mediante la identificación de factores naturales o antropogénicos asociados (Molina \& Darwich 2011). De ahí que, el análisis de los informes de morbilidad y mortalidad de estas aves de vida, remitidas a los centros de rescate o zoológicos, ha brindado información sobre las causas primarias y secundarias, así como en la evaluación del estado de salud de las poblaciones silvestres, por medio de los datos que se pueden obtener a partir de los individuos, como por ejemplo biometría, valores fisiológicos o presencia de parásitos. (Fix \& Barrows 1990; Morishita et al. 1998; Wendell et al. 2002; Molina et al. 2011).

Por otra parte, en relación con la causalidad de búhos de vida libre a los centros de rescate y zoológicos, se reporta mayormente traumas, orfandad, intoxicaciones, electrocuciones y causas no determinadas, las cuales están relacionadas principalmente con actividades humanas (choque contra vehículos, edificios o ventanas, lesiones por armas de fuego y líneas de alta tensión) (Fix \& Barrows 1990; Deem et al. 1998; Morishita et al. 1998, Wendell 2002; Samour 2004; Komnenou et al. 2005; Rodríguez et al. 2010; Molina et al. 2011; Montes de Oca 2016), además, la mayoría de estos estudios se han realizado en Norteamérica y Europa. Sin embargo, para Costa Rica no existen reportes de causas de morbilidad/ mortalidad en a centros de recate y zoológicos con este grupo de rapaces.

Cabe considerar por otra parte, que cualquier información sobre de estas especies es significativa, ya que son consideradas valiosas centinelas de los cambios ambientales, debido a su posición en la parte superior de la cadena alimenticia (Molina et al. 2011), ya que a partir de la ingesta de presas envenenadas accidental o intencionadamente, las aves rapaces pueden intoxicarse de forma primaria $\mathrm{o}$ secundaria, esto porque las aves son más sensibles que otros vertebrados (Robles et al. 2007), de manera que cuando cualquier eslabón de la cadena alimenticia se elimine del ecosistema, tendrá un efecto en cascada sobre la estabilidad del ecosistema y el funcionamiento del que depende la humanidad (Hunter \& Hutchinson 1994; Pimm et al. 1995; Brooks et al. 2006).

De modo que, estudios a largo plazo proporcionan información útil para la conservación de las rapaces (Harris \& Sleeman 2007; Rodríguez et al. 2010; Molina et al. 2011; Montes de Oca et al. 2016).

Los objetivos de estudio fueron describir las causas principales de ingreso para las especies del Orden Strigiformes en el Parque Zoológico y Jardín Botánico Nacional Simón Bolívar (ZJBSB), así como resumir los diagnósticos clínicos primarios, localidades de procedencia y especies ingresadas, utilizando la información generada en las hojas de admisión durante un período de 20 años (1995-2015).

\section{Metodología}

La recopilación de información se realizó en el ZJBSB. Se utilizaron las hojas de registro de animales que fueron ingresados durante 1995 hasta 2015.

De las hojas de ingreso se seleccionaron todas especies del orden Strigiformes y se extrajeron los siguientes datos: fecha de ingreso, especie, localidad y causa de admisión, adicionalmente, se recopiló información basados en: (a) el examen físico realizado por el veterinario en la instancia de admisión; (b) la historia registrada por el rescatista que encontró el ave; (c) reportes de resultados de pruebas diagnósticas complementarias cuando estaban disponibles (por ej.: radiografías). 
Posteriormente, se elaboró una lista con la cantidad de individuos por especie. Así mismo, se agruparon según la provincia de la cual procedían y seguidamente, se categorizaron las causas de admisión.

Es importante recalcar que una gran cantidad de los registros de los años noventa y principios de la década del 2000, carecía de la información sobre el destino final de los animales, pues eran solamente hojas de ingreso, por lo que se decidió omitir esta información y enfocarse en las causas de admisión. Sin embargo, a partir del 2007, se tienen datos de muchos individuos que recibieron cirugía ortopédica y su destino, este número corresponde a 58 pacientes, de los cuales se obtuvieron los porcentajes de éxito y destino posterior a la cirugía.

Las causas de admisión se categorizaron de la siguiente manera: (1) trauma, (2) huérfanos, (3) intoxicación, (4) electrocución, (5) causas desconocidas (cuando no se obtuvo un diagnóstico definitivo). Por ejemplo, en la categoría de trauma incluimos todas las aves que presentaron fracturas; en el caso de las aves envenenadas se identificaron usando síntomas externos (incapacidad para volar, rigidez del cuello, dilatación pupilar).

Luego, la categoría por trauma se clasificó según el tipo de lesión, de la siguiente manera: (1) fractura en extremidades (anteriores o posteriores), (2) trauma cráneo encefálico (TCE), (3) Heridas en piel y tegumentos. De las 140 fracturas de alas, 87 tenían la información sobre el hueso afectado, y de las 8 de miembros posteriores solamente el $75 \%$ contaban con esa información.

De todos los datos se obtuvo el porcentaje excepto para la abundancia de las especies, la cual se presenta en números absolutos. La estadística utilizada fue descriptiva y para realizar los gráficos se utilizó el software de Microsoft Excel ${ }^{\circledR} 2010$.

\section{Resultados}

Un total de 352 individuos pertenecientes a nueve especies de Strigiformes, se registraron de en el ZJBSB, durante los años 1995 al 2015. La especie más abundante fue la Lechucita Neotropical (Megascops choliba) con 141 individuos (40.05\%), mientras que la Lechucita Serranera (Megascops clarkii) y el Búho Penachudo (Lophostrix cristata) fueron los menos abundantes, con cuatro individuos cada uno, 1.13 \% (Cuadro 1).

De acuerdo con la información recopilada de las hojas de admisión el mayor porcentaje de individuos provino de las provincias de San José $(80,91 \%, n=285)$ y Heredia $(6,57 \%, n=23)$, mientras que los menos frecuentes de Guanacaste $(0.51 \%, n=2)$ y Puntarenas $(1,01 \%, n=3)$, se registraron, también individuos de Cartago (4,55 \%, $\mathrm{n}=16)$, Alajuela $(3,54 \%, \mathrm{n}=12)$ y Limón $(3.03 \%, \mathrm{n}=11)$. Así pues, los ingresos de estas aves al ZJBSB, provinieron de todo el país.

En cuanto a la dinámica de las admisiones de individuos por año, en general se mostró relativamente homogénea a lo largo del periodo de estudio, en un rango de 10 a 20 individuos, excepto los años 1999 $(\mathrm{n}=35)$ y $2000(\mathrm{n}=48)$ (Figura 1). 
Cuadro 1. Especies e individuos del orden Strigiformes admitidas en el Parque Zoológico y Jardín Botánico Nacional Simón Bolívar durante 1995-2015.

\begin{tabular}{ll}
\hline Especie & N. $^{\circ}$ de Aves (\%) \\
\hline Lechucita Neotropical (Megascops choliba) & $141(40.05 \%)$ \\
Mochuelo Común (Glaucidium brasilianum) & $63(17.89 \%)$ \\
Búho Listado (Pseudoscops clamator) & $53(15.05 \%)$ \\
Lechuza Café (Ciccaba virgata) & $35(9.94 \%)$ \\
Lechuza Ratonera (Tyto alba) & $32(9.09 \%)$ \\
Búho de Anteojos (Pulsatrix perspicillata) & $14(3.97 \%)$ \\
Lechuza Blanco y Negro (Ciccaba nigrolineata) & $7(1.98 \%)$ \\
Lechucita Serranera (Megascops clarkii) & $4(1.13 \%)$ \\
Búho Penachudo (Lophostrix cristata) & $4(1.13 \%)$ \\
Total & $\mathbf{3 5 2 ( 1 0 0 \% )}$ \\
\hline
\end{tabular}

Fuente: elaboración propia.

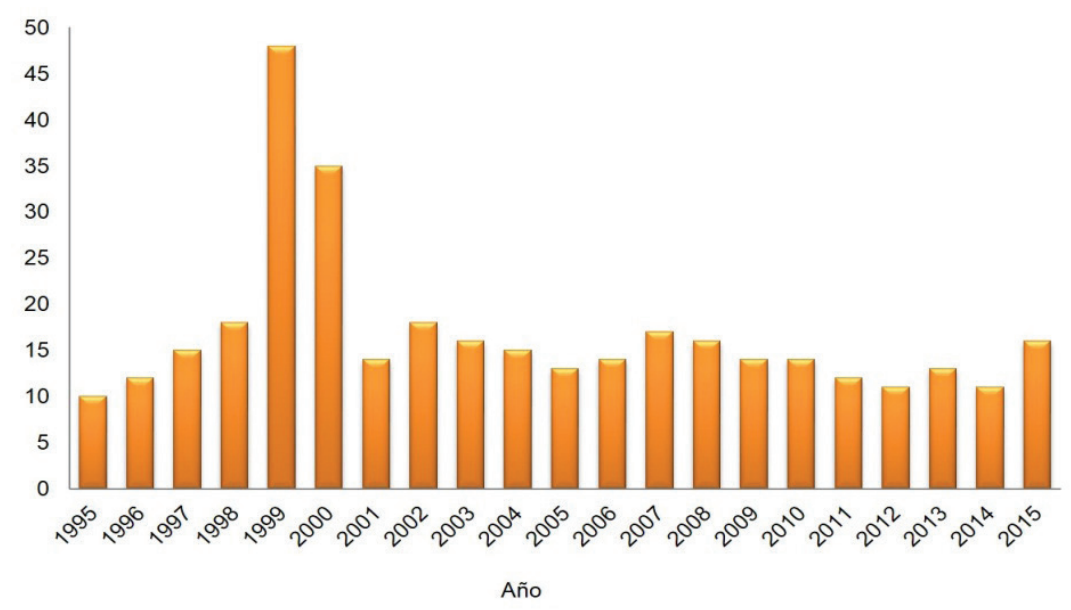

Figura 1. Admisiones del Orden Strigiformes distribuidas anualmente a lo largo del periodo de 1995 a 2015.

Fuente: elaboración propia.

Por otra parte, el trauma fue la causa de admisión más frecuente. De las 352 aves admitidas el $54 \%$ $(\mathrm{n}=190)$ presentaba lesiones originadas por esta causa Desafortunadamente, la causa que originó el trauma era desconocida para prácticamente todos los casos registrados. La segunda causa de ingreso fueron los huérfanos con un $38 \%(n=134)$ (Figura 2). 


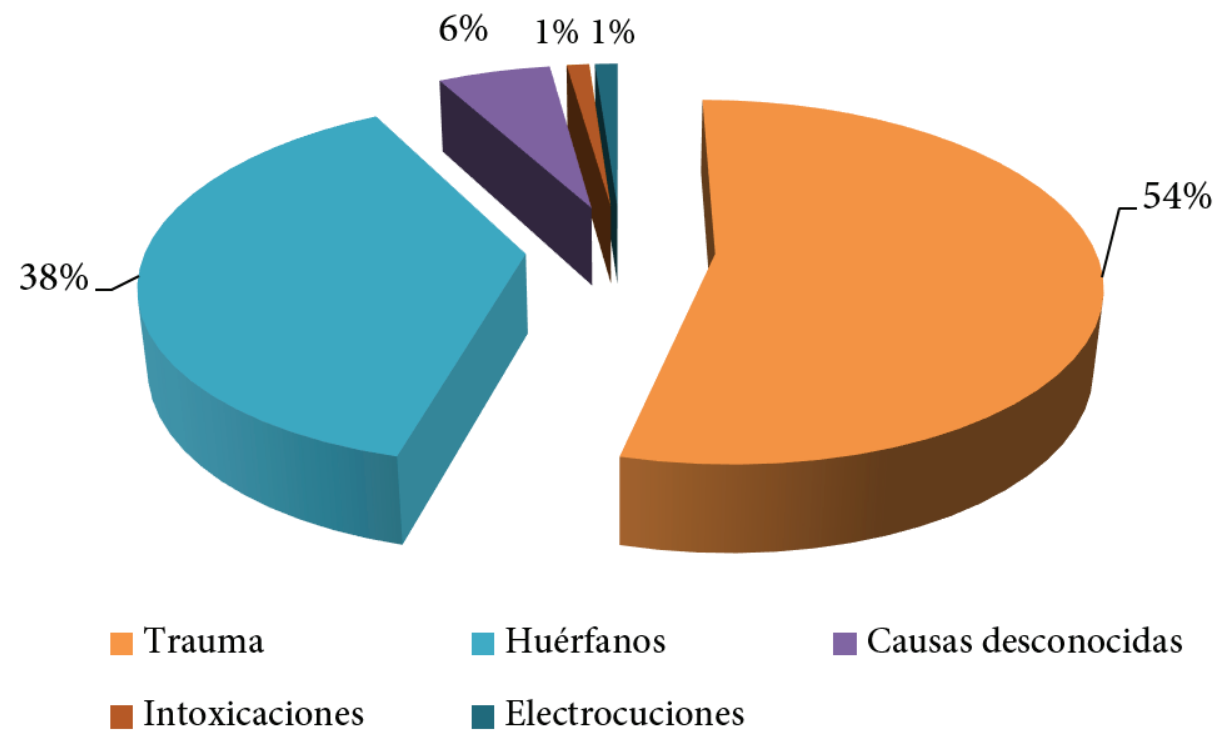

Figura 2. Causas de admisión para el Orden Strigiformes, en el Parque Zoológico y Jardín Botánico Nacional Simón Bolívar durante 1995 al 2015. Fuente: elaboración propia.

Ahora bien, de los 190 (54 \%) individuos admitidos por trauma, el $78 \%(\mathrm{n}=148)$ representa las fracturas al ser esta causa el tipo de lesión más frecuente, de modo que, del total de las fracturas encontradas, el $93 \%(n=140)$ fueron en los miembros anteriores. En 87 de los individuos registrados se indicaba el hueso fracturado, al ser el húmero el hueso más afectado con $76 \%(\mathrm{n}=66)$, seguido de ulna y radio con $22 \%(n=19)$ y metacarpos $2 \%(n=2)$ (Fig. 3$)$ y el $7 \%(n=8)$ en los miembros posteriores. En 6 de los individuos se indicaba el hueso afectado, donde el mayormente involucrado fue el tibiotarso $67 \%(n=4)$ y en segundo lugar el fémur $33 \%(n=2)$.

En segundo lugar, del total de la categoría de trauma, se encontró Trauma Cráneo Encefálico (TCE) con $16 \%(\mathrm{n}=30)$, y por último heridas de piel solo un $6 \%(\mathrm{n}=11)$. 


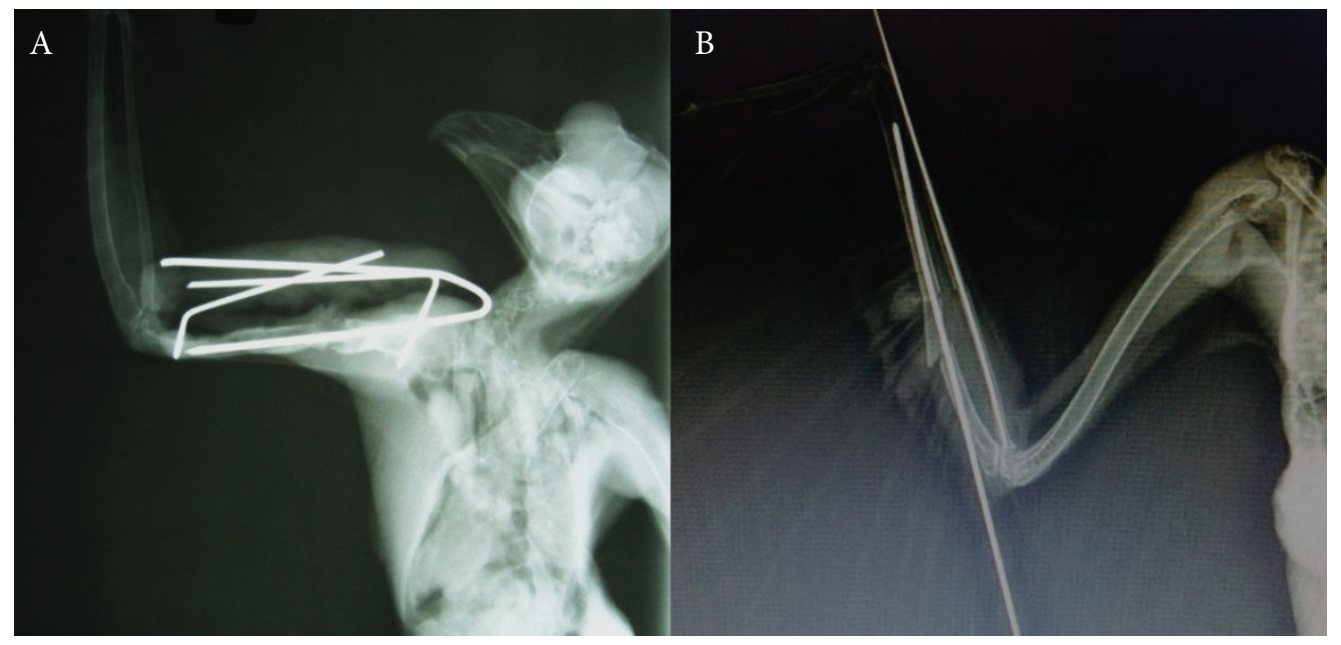

Figura 3. Radiografías postquirúrgicas en miembros anteriores de Strigiformes. (A) Fractura de húmero en Lechuza Blanco y Negro (Ciccaba nigrolineata). (B) Fractura de radio y ulna en Búho de Anteojos (Pulsatrix perspicillata)

Fuente: elaboración propia.

\section{Discusión}

Costa Rica posee 17 especies registradas del Orden Strigiformes pertenecientes a dos familias: Tytonidae con una especie y Strigidae con los 16 restantes (Stiles et al. 2003; Garrigues et al. 2016), en este estudio se ven representadas una buena parte de las especies registradas para Costa Rica (nueve especies).

No obstante, en el presente estudio podría haber un sesgo en las especies que han sido registradas, debido a que las aves que fueron admitidas mayormente son las que naturalmente ocurren cerca de asentamientos humanos (Obando et al. 2014), por lo cual es más probable, no solo que ocurran accidentes de origen antropogénico, sino de que tengan la posibilidad de ser encontradas por personas. Más del $80 \%$ de los ingresos fueron de la provincia de San José, al ser de los cantones cercanos al Zoológico y con mayor población (Cantón Central 26 \%, Montes de Oca $13 \%$, Desamparados, Moravia y Aserrí 11 \% cada uno, Goicoechea 7 \%, Vásquez de Coronado 6 \%, Escazú $5 \%$, Santa Ana y Curridabat 3 \% cada uno y Tibás y Alajuelita $2 \%$ cada uno).

Así también, las especies que más llegaron durante el estudio fueron Lechucita Neotropical (M. choliba), el Mochuelo Común (G. brasilianum), el Búho Listado (P. clamator), la Lechuza Café (C. virgata) y la Lechuza Ratonera (T. alba), esto posiblemente se debió a que estas especies se encuentran ampliamente distribuidas en el valle central y cerca de asentamientos urbanos, como jardines, cafetales, potreros y edificaciones como el caso de la lechuza ratonera (Stiles et al. 2007, Camacho-Varela 2014, Garrigues 2014,). Por lo tanto, esta situación hace suponer, que estos individuos en su mayoría sean llevados a centros de rescate o zoológicos cercanos al lugar donde fueron encontrados, tanto los polluelos hallados en el suelo, así como animales adultos afectados. 
Al estar estas especies mayormente asociados a ambientes humanos, la probabilidad de tener accidentes es alta, debido al tráfico vehicular, construcción de edificios, torres de transmisión y otras edificaciones y esto puede relacionarse con las diferentes causas de ingreso (Rodríguez et al. 2010; Molina et al. 2011).

Los datos revelan que el $54 \%$ de los búhos que ingresaron a consulta, la causa principal fue algún tipo de trauma. Estudios similares en donde se logró identificar las causa de las lesiones incluyen colisiones contra vehículos, ventanas, alambres de púas o disparos (Fix \& Barrows 1990; Morishita et al. 1998; Komnenou et al. 2005; Rodríguez et al. 2010; Molina et al. 2011), sin embargo, en este estudio las causas del trauma no fueron determinadas, porque la mayoría de los animales solo fueron encontrados con la lesión, y remitidos con varios días posterior al accidente, lo cual se determinó por la apariencia de la lesión, sin poder asociar un origen específico. No obstante, podemos presumir que las causas son similares a las de los estudios mencionados en la literatura.

Un alto porcentaje de los ingresos por trauma son fracturas (78 \%), y de estas un $93 \%$ en las extremidades anteriores (alas), al ser el húmero el hueso mayormente involucrado. El húmero es un hueso hueco en aves, y se expone con mucha frecuencia, debido a que casi un tercio de la diáfisis no tiene masa muscular que lo proteja (Redig \& Ponder 2016). Las fracturas en alas son comunes por disparos, o cuando el ala choca con un cable o por atropellos, la cual es una probabilidad muy alta en el caso de búhos (Yoon et al. 2008).

Los búhos que ingresaron con fracturas de alas fueron tratados con cirugía ortopédica cuando la lesión así lo permitió, ya que en muchos casos los tejidos blandos estaban muy lesionados, además en muchas ocasiones el ave fue traída días después que ocurriera la fractura, presentando exposición (especialmente del húmero) de uno o varios fragmentos óseos, con consecuente pérdida de vascularidad o infección (Redig \& Ponder 2016), por lo que muchos de estos casos fueron sometidos a una eutanasia humanitaria, y en otros aunque la fractura pudo ser corregida, el ala quedó con limitaciones biomecánicas para el vuelo y limitó la subsecuente liberación.

De los casos sometidos a procedimiento quirúrgico $(n=58)$, un $54 \%$ se liberó, el restante porcentaje (46\%), aunque fue exitosa la osteosíntesis, quedó con una movilidad disminuida, limitada o con una reducción en la función biomecánica, para efectuar el vuelo.

Por otra parte, los choque contra vidrios o carros generalmente ocasionan TCE, con lesiones o traumas oculares, al colisionar de frente contra el objeto (Cousquer 2005; Seruca et al. 2012); sin embargo, ninguno de los individuos que ingresaron con TCE (16\%) presentaba fracturas. El tratamiento para estos casos incluyo analgésicos no esteroideos, terapia de fluidos al ser ingresados, entre otros; y posteriormente a su estabilización, se trataron las lesiones oculares mayormente asociadas a lesiones corneales y uveítis.

La segunda causa de ingreso al ZNJBSB fue aves huérfanas (38\% $\mathrm{n}=134)$, estos resultados son similares a los reportados por Wendell et al. 2002, Komnenou et al. 2005; Molina-López et al. 2011 y Montes de Oca et al. 2016. Estos autores reportan a la orfandad como la segunda causa de admisión de rapaces de vida libre en los centros de rescate. Esto se debe a que la mayoría de los animales en esta condición son llevados a los centros de rescate, principalmente porque las personas con buenas intenciones a menudo confunden a los polluelos (con capacidad de volar) con aves huérfanas (Komnenou et al. 2005), o los encuentran en sus nidos en el caso de especies que naturalmente nidifican en el suelo (Obando et al. 
Rev. Ciencias Veterinarias, Vol. 37, Nº 1, [1-11], E-ISSN: 2215-4507, enero-junio, 2019

DOI: http://dx.doi.org/10.15359/rcv.37-1.1

Ciencias

URL: http://www.revistas.una.ac.cr/index.php/veterinaria/index

2014), y estas podrían ser las mismas causas de admisión para este grupo de aves en el ZNJBSB. Cabe añadir que la mayor parte de los animales huérfanos admitidos, se quedan en exhibición con fines de conservación, educativos o científicos, principalmente por que presentan impronta conductual con humanos.

Recapitulando, determinar las causas de accidentes en poblaciones de aves rapaces silvestres es difícil, porque la toma de datos es imparcial y rara vez es posible obtener una información completa y corroborable, tanto por la que es brindada por las personas que encuentran el ave, como por el hecho de que los datos son tomados por diferentes personas dentro de las instituciones y, aunque la causa inmediata de enfermedad o muerte puede ser diagnosticada con precisión al momento del ingreso, las condiciones subyacentes que originaron las lesiones o la muerte, a menudo no pueden ser establecidas (Wendell et al. 2002). Sin embargo, las causas de admisión de Strigiformes presentadas en las instalaciones de centros de rescate y zoológicos, pueden proporcionar una idea de las situaciones que podrían estar afectando a las poblaciones silvestres. Al ser plenamente conscientes del nivel de sesgos que arrastran estos datos (Deem et al. 1998 y Rodríguez et al. 2010).

En conclusión, para este estudio, las causas de ingreso más importantes fueron las fracturas de los miembros anteriores (alas) y los animales huérfanos de especies de búhos, que están presentes en los cantones más poblados de la provincia de San José; por lo que se considera que los estudios retrospectivos pueden convertirse en una herramienta útil para que las instituciones que admiten aves silvestres, puedan atender de mejor manera este tipo de casos, con el fin de obtener éxito en la sobrevivencia y posible liberación de los animales afectados.

\section{Conflicto de intereses}

Los autores declaran que no existe conflicto de intereses.

\section{Referencias}

Brooks, T., Mittermeier, R., Da Fonseca, G., Gerlach, J., Hoffmann, M., Lamoureux, J., Mittermeier, C., Pilgrim, J. \& Rodrigues, A. 2006. Global biodiversity conservation priorities. Science 313: 58-61. Doi: $10.1126 /$ science.1127609

Camacho-Varela, P. 2014. ¿Cómo identificar correctamente los búhos Megascops presentes en Costa Rica? Zeledonia 18(2): 3-15.

Cousquer, G. 2005. Ophthalmological findings in free-living tawny owls (Strix aluco) examined at a wildlife veterinary hospital. Vet Rec 156(23): 734-739. Doi: https://doi.org/10.1136/vr.156.23.734

Deem, S., Terrell, S. \& Forrester, D. 1998. A retrospective study of morbidity and mortality of raptors in Florida: 1988-1994. J Zoo Wildlife Med 29(2): 160-164.

Fix, A.S. \& Barrows, S.Z. 1990. Raptors Rehabilitates in Iowa during 1988 and 1987: a Retrospective Study. J Wildlife Dis 26(1):18-21. Doi: https://doi.org/10.7589/0090-3558-26.1.18 
Garrigues, R. \& Dean, R. 2014. The birds of Costa Rica: A field guide. Zona Tropical, Ithaca, Nueva York.

Garrigues, R., Araya-Salas, M., Camacho-Varela, P., Montoya, M., Obando-Calderón, G. \& RamírezAlán, O. 2016. Lista Oficial de las Aves de Costa Rica: Actualización 2016. Zeledonia 20 (2): 3-12.

Harris, M. \& Sleeman, J. 2007. Morbidity and mortality of bald eagles (Haliaeetus leucocephalus) and peregrine falcons (Falco peregrinus) admitted to the Wildlife Center of Virginia, 1993-2003. J Zoo Wildlife Med 38(1): 62-66. Doi: https://doi.org/10.1638/05-099.1

Hunter, Jr.M. \& Hutchinson, A. 1994. The virtues and shortcomings of Parochialism: Conserving species that are locally rare, but globally common. Conserv Biol 8(4): 1163-1165. Doi: https://doi. org/10.1046/j.1523-1739.1994.08041163.x

Komnenou, A., Georgopoulou, I., Savvas, I. \& Dessiris, A. 2005. A retrospective study of presentation, treatment, and outcome of free-ranging raptors in Greece (1997-2000). J Zoo Wildlife Med 36(2): 222-228. https://doi.org/10.1638/04-061.1

Molina-López, R.A. \& Darwich, L. 2011. Causes of admission of little owl (Athene noctua) at a wildlife rehabilitation centre in Catalonia (Spain) from 1995 to 2010. Anim Biodiv Conserv 34(2): 401-405.

Molina-López, R.A., Casal, J. \& Darwich, L. 2011. Causes of morbidity in wild raptor populations admitted at a wildlife rehabilitation centre in Spain from 1995-2007: A long term retrospective study. PLOS ONE 6(9): e24603. Doi: 10.1371/journal.pone.0024603

Montes de Oca, N., Calabuig, P., Corbera, J.A. \& Orós, J. 2016. Causes of admission for raptors to the Tafira Wildlife Rehabilitation Center, Gran Canaria Island, Spain: 2003-2013. J Wildlife Dis 52(3): 647-652. Doi: 10.7589/2015-09-255.

Morishita, T., Fullerton, A., Lownestine, L., Gardner, I. \& Brooks,D. 1998. Morbidity and mortality in free-living raptorial birds of Northern California: A retrospective study, 1983-1994. J Avian Med Surg 12(2): 78-81.

Obando-Calderón, G., Acosta-Chaves, V., Camacho, P., Elizondo, P., Montoya, M., Oviedo, S. \& Saénz, Y. 2014. Aves en problemas: Las causas, cuándo y cómo ayudarlas. Zeledonia 18(1): 28-53.

Pimm, S.L., Russell, G.L., Gittleman, J.L. \& Brooks, T.M. 1995. The future of biodiversity. Science 269: 347-350. Doi: https://doi.org/10.1126/science.269.5222.347

Robles Sanmartín, J., Hernández Vicente, T. \& Luque Díez, G. 2007. Tratamiento de la intoxicación por organofosforados en aves rapaces. Rev. Complutense de Ciencias Veterinarias. 1(2): 424-437.

Rodríguez, B., Rodríguez, A., Siverio, F. \& Siverio, M. 2010. Causes of raptor admissions to a wildlife rehabilitation center in Tenerife (Canary Islands). J Raptor Res 44(1): 30-39. Doi: https://doi. org/10.3356/JRR-09-40.1

Redig, P.T \& Ponder, J. 2016. Orthopedic surgery. En: Samour, J (Ed). Avian Medicine. Mosby International Ltd, Estado Unidos, p. 312-358.

Naldo, J.L \& Samour, J.H. 2004. Causes of morbidity and mortality in Falcons in Saudi Arabia. J Avian Med Surg 18(4): 229-241. Doi: https://doi.org/10.1647/2002-013 
Seruca, C., Molina-López, R., Peña, T., \& Leiva, M. 2012. Ocular consequences of blunt trauma in two species of nocturnal raptors (Athene noctua and Otus scops). Vet Ophthalmol 15(4): 236-244. Doi: https://doi.org/10.1111/j.1463-5224.2011.00976.x

Stiles, F.G., Skutch, A.F. \& Gardner, D. (Ed). 2007. Guía de aves de Costa Rica. INBio, Santo Domingo, Heredia, p. 218-227.

Yoon, H.Y., Fox, D.B., \& Jeong, S-w. 2008. Long bone fractures in raptors: 28 cases (2004-2007). J Veterinary Clinics. 25(3): 215-217.

Wendell, M.D, Sleeman J.M. \& Kratz, G. 2002. Retrospective study of morbidity and mortality of raptors admitted to Colorado State University Veterinary Teaching Hospital during 1995 to 1998. J Wildlife Dis 38(1): 101-106. Doi: https://doi.org/10.7589/0090-3558-38.1.101 